EPJ Web of Conferences 110,01029 (2016)

DOI: $10.1051 /$ epjconf/201611001029

(C) Owned by the authors, published by EDP Sciences, 2016

\title{
DIFFUSION COEFFICIENT WHEN FINE AEROSOL MEDIA PROPAGATE IN A CONFINED VOLUME
}

\author{
N.V. Korovina ${ }^{1}$, I.K. Zharova, a , O.B. Kudryashova ${ }^{1}$, S.S. Titov ${ }^{1}$ \\ ${ }^{1}$ Institute for Problems of Chemical and Energetic Technologies, Siberian Branch of the Russian Academy of Sciences (IPCET SB RAS), Biysk, \\ Russia \\ ${ }^{2}$ Scientific Research Institute of Applied Mathematics and Mechanics, National Research Tomsk State University, Tomsk, RussiaTomsk Polytechnic \\ University, Tomsk, Russia
}

\begin{abstract}
An experimental estimation is reported of the value of the effective diffusion coefficient during aerosol deposition in a confined volume. Aerosol propagation regularities have experimentally been studied in a chamber of a complex configuration with different placement of aerosol generators.
\end{abstract}

\section{Introduction}

The necessity of solving problems of aerosol media propagation in confined spaces emerges from the development of degassing technologies and design of non-lethal devices for suppression of terrorist elements, rectification of the consequences of man-made disasters conjugated with emission of toxic agents, as well as for creation of new fire-extinguishing equipment and modernization of the existing one, etc. Aerosols are broadly applied as a disinfectant and an antibacterial. For functioning and advancement of many industrial sectors, it is important to take cognizance of phenomena and processes that involve aerodispersed systems. In this respect, the study into regularities of aerosol formation dynamics and propagation is topical.

The shock-wave technique for the generation of aerosols offers a range of advantages [1], the major of which is the possibility to produce a high-dispersion aerosol. Among the merits of this technique is also its self-sufficiency and fast action in generating an aerosol medium $(\sim 1 \mathrm{~ms})$ at a speed of the atomized medium outflow of 100-300 m/s. However, despite demands in practical applications, the propagation mechanisms of fine aerosols being thus obtained are nowadays studied not enough. In particular, there is no reliable data on basic parameters of aerosol propagation within confined spaces.

The present work outlines the results of the experimental determination of the value of the effective diffusion coefficient, $D$, when an aerosol is deposited in a confined volume of different spatial configurations.

\section{Physico-mathematical model of liquid-droplet aerosol propagation}

An analysis of the results of the preliminary experiments with process visualization has shown that, by the shock-wave aerosol generation in a confined volume, a primary cloud is formed whose shape is close to a spherical one (Fig .1). The characteristic time of the primary cloud formation is about $200 \mathrm{~ms}$; the primary cloud radius is considerably lower than the characteristic dimension of the confined volume. Thence, it was supposed that the aerosol particles generated by the shock-wave technique propagate within the confined volume in two stages: formation of the primary aerosol cloud and its evolution due to diffusion and gravitational sedimentation of the particles [2].

\footnotetext{
${ }^{a}$ Corresponding author: zharova@niipmm.tsu.ru
} 

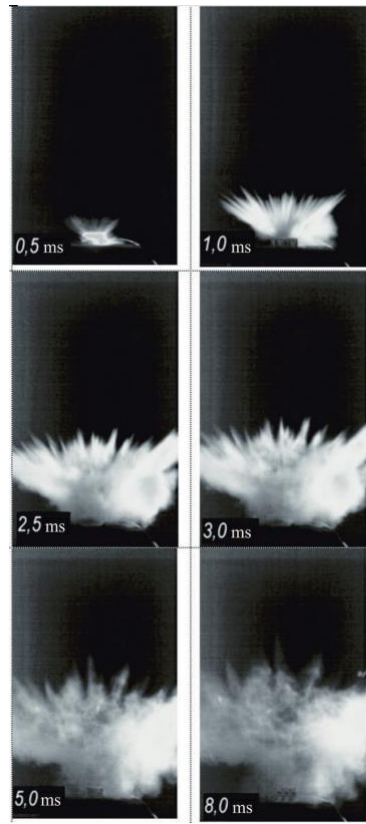

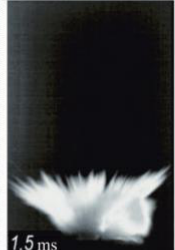

$1,5 \mathrm{n}$

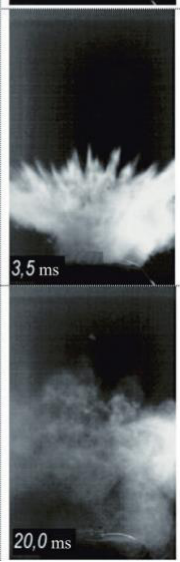

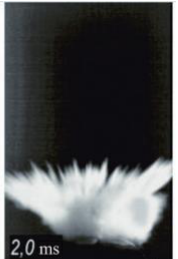

$2,0 \mathrm{~ms}$

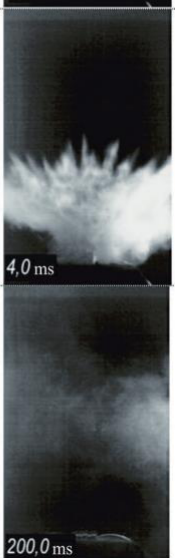

Figure 1. Video frames of the shock-wave atomization of liquid.

In the framework of the trajectory approach, the aerosol diffusion process is mathematically described by the equation:

$$
\frac{\partial c}{\partial t}=\frac{D}{r^{2}} \frac{\partial}{\partial r}\left(r^{2} \frac{\partial c}{\partial r}\right)
$$

with initial and boundary conditions:

$$
\begin{gathered}
t=0: c=\left\{\begin{array}{l}
c_{0}, r \leq r_{0} ; \\
0, r>r_{0} ;
\end{array}\right. \\
r=0: \frac{\partial c}{\partial r}=0 ; \\
r \rightarrow \infty: c=0,
\end{gathered}
$$

where $r_{0}$ - the radius of the primary (spherical) aerosol cloud; $c_{0}=\frac{3 m_{0}}{4 \pi r_{0}^{3}}-$ the initial concentration of the atomized matter with the weight $m_{0}$ in the primary cloud; $D$ - the diffusion coefficient.

The analytical solution of the problem (1)-(4) for the mass fraction $C(r, t)$ of the atomized matter is written as [1]:

$$
\begin{aligned}
& C(r, t)=\frac{1}{2}\left\{\operatorname{erfc}\left(\frac{r-r_{0}}{2 \sqrt{D t}}\right)-\frac{2}{r} \sqrt{\frac{D t}{\pi}} \exp \left[-\frac{\left(r-r_{0}\right)^{2}}{4 D t}\right]-\right. \\
& \left.-\operatorname{erfc}\left(\frac{r+r_{0}}{2 \sqrt{D t}}\right)+\frac{2}{r} \sqrt{\frac{D t}{\pi}} \exp \left[-\frac{\left(r+r_{0}\right)^{2}}{4 D t}\right]\right\}
\end{aligned}
$$

\section{Experimental determination of diffusion coefficient}

The experiments to estimate the diffusion coefficient of a polydisperse liquid-droplet aerosol (particle radius $r p \leq 10 \mu \mathrm{m}$ ) have been performed on a stand equipped with an optical diagnostics system. A schematic representation and a general view of the experimental stand are illustrated in Figures 2 and 3. 


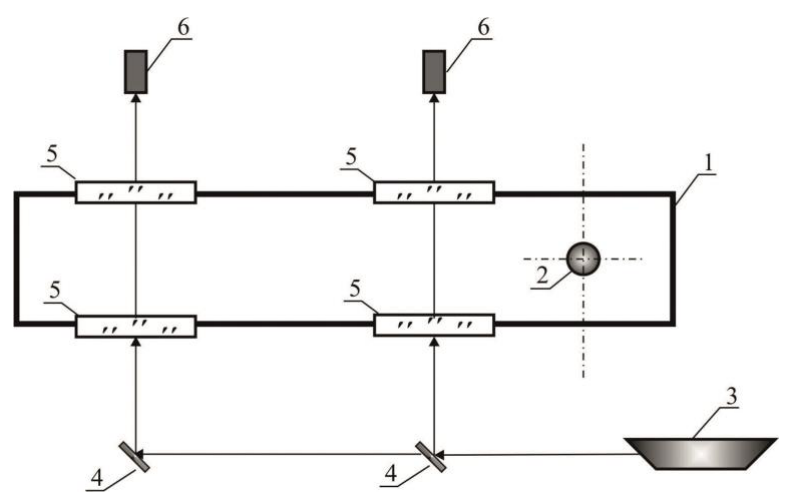

Figure 2. A schematic of the experimental stand: 1 - chamber, 2 - shock-wave generator, 3 - probe laser, 4 - array of mirrors, 5 - optical windows, 6 - radiation detector.

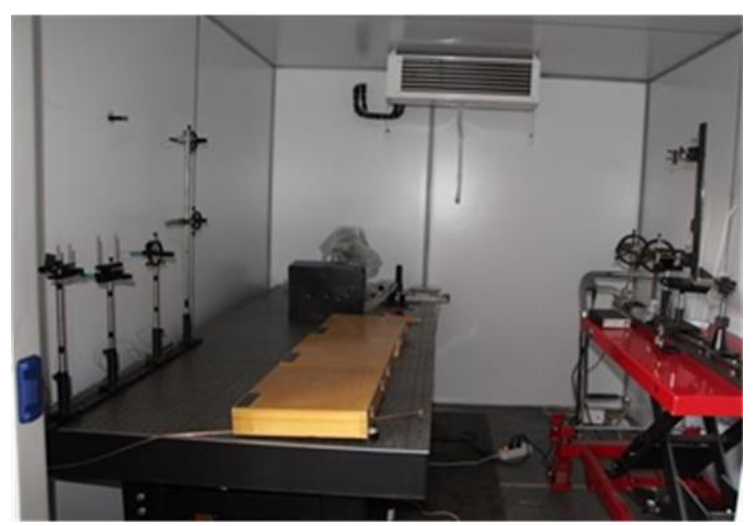

Figure 3. A general view of the experimental stand.

The spray pattern and the aerosol cloud were visualized by high-speed video recording. The concentration and dispersity of the particles of the atomized liquid was quantified using contactless optical methods. The aerosol particle concentration was measured by the spectral transparency method [3]. The particle dispersity was determined by the method of small-angles of scattering indicatrix [4].

The effective value of the diffusion coefficient of fine aerosol droplets during the evolution of the aerosol cloud in the confined volume was determined through the minimization of the functional built from the experimental data and $C(r, t)$ values calculated by eq. (5). The resultant value was $D=1.2 \cdot 10-3 \mathrm{~m}^{2} / \mathrm{s}$, which is two orders of magnitude greater than the molecular diffusion coefficient. Having regard to the diffusion coefficient value obtained, the concentration range upon the shock-wave atomization of a $20 \%$ aqueous glycerol was determined on the grounds of numerically solving the problem (1)-(4). Figure 4 displays a comparison between the mass particle concentrations measured by the spectral transparency method and those calculated with account of the effective diffusion coefficient value obtained. 


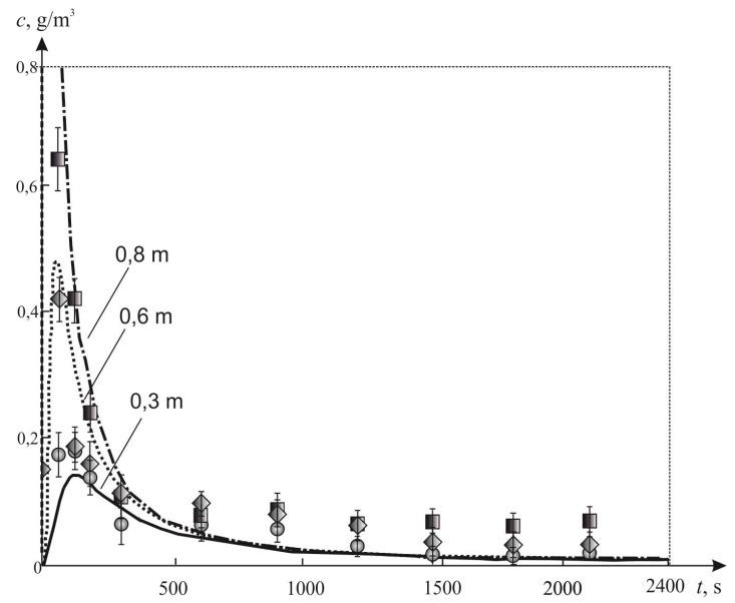

Figure 4. The particle concentration plotted against time at different distances from the aerosol generators: $\bullet, \bullet, \mathbf{\square}-$ experiment; ---, $\bullet \bullet,--\bullet--\bullet$ -- - calculation.

Taking into account the information acquired, the aerosol propagation was experimentally investigated in a $\Gamma$-shaped chamber of a horizontal (Fig. 5) and a vertical (Fig. 6) configurations.

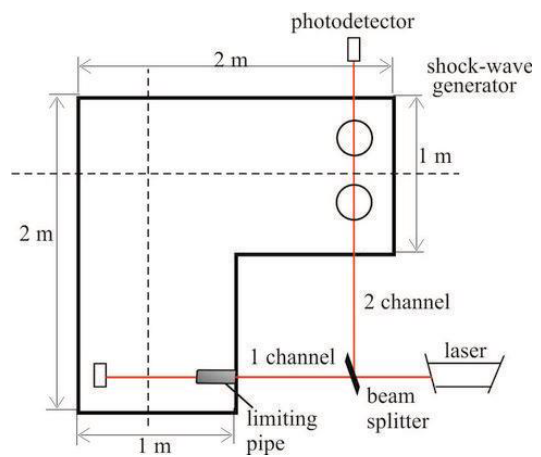

a)

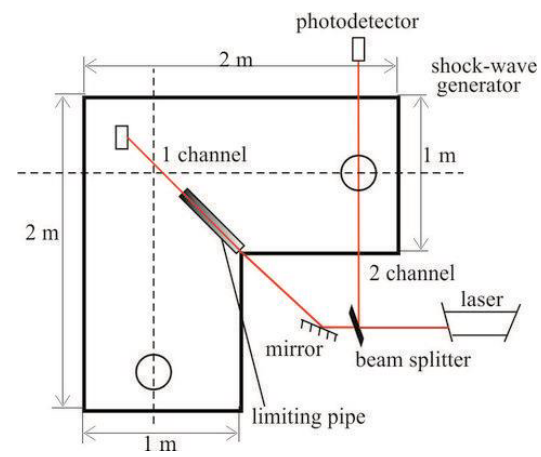

b)

Figure 5. A schematic of the experimental setup of the horizontal configuration with the generators positioned in one (a) and different (b) places of the confined volume.

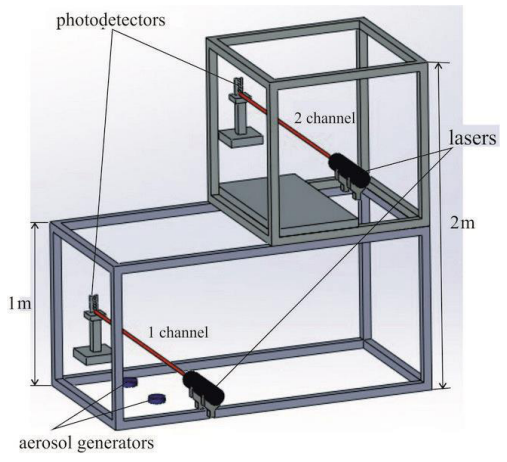

a)

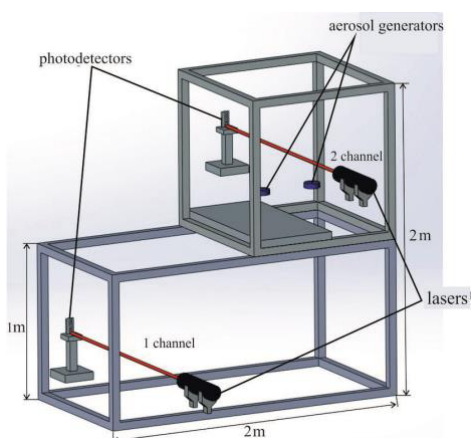

b)

Figure 6. A schematic of the experimental setup of the vertical configuration with the generators positioned at the bottom (a) and top (b) of the confined volume. 
The aerosol characteristics in the experiments were measured by a modified setup [5]-a two-channel variant of the LID-2M laser instrument (Fig. 7)-which enables simultaneous measurement of dispersion parameters in different cross-sections of the confined volume.

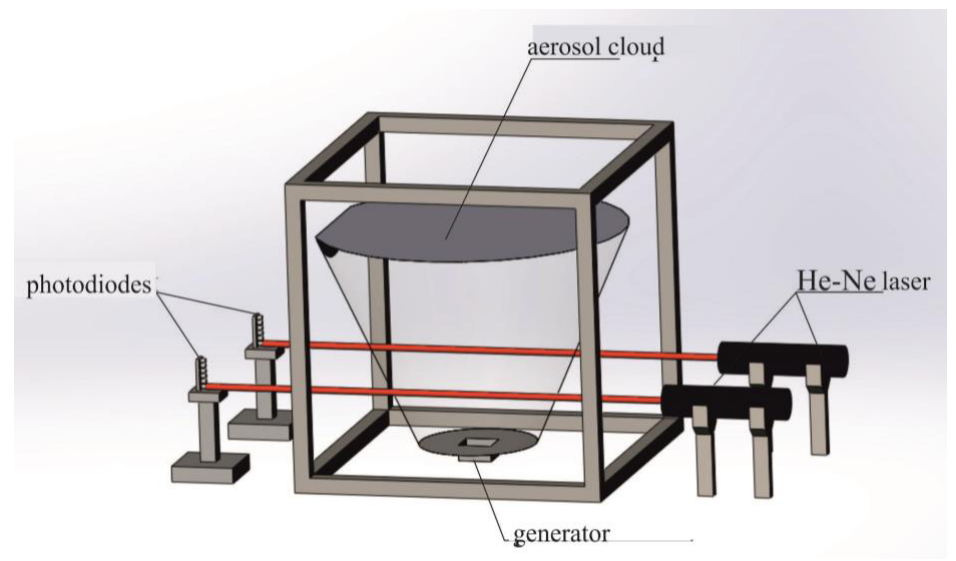

Figure 7. A two-channel variant of the LID-2M laser instrument.

In the horizontally configured volume when the generators were positioned in a concentrated manner (Fig. 5a), the first channel of the laser instrument was used to measure aerosol characteristics directly above the generators, while the second channel was used for the measurement at a distance of $\sim 170 \mathrm{~cm}$ from the generators. In case the aerosol generators were positioned in the opposite parts of the confined volume (Fig. 5b), the first channel of the laser instrument measured aerosol characteristics directly above one of the aerosol generators, while the second channel measured aerosol cloud characteristics at the same (maximum) distance from both generators.

In the vertically configured volume when the generators were positioned at the bottom of the confined volume (Fig. 6a), the measurements were run directly above the aerosol generators by using the first channel, and at the top of the volume by using the second channel. When the generators were located at the top of the $\Gamma$-shaped space (Fig. $6 \mathrm{~b}$ ), aerosol characteristics were measured in both parts of the confined volume.

\section{Results of experimental study}

Figure 8 shows the results of the experimental and calculational study when the fine aerosol propagates in the horizontally configured $\Gamma$-shaped chamber.

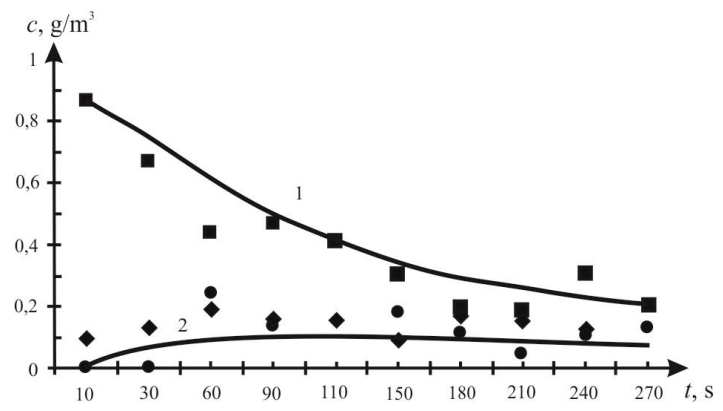

Figure 8. The particle concentration versus time upon propagation in the horizontally configured $\Gamma$-shaped chamber: 1,2 - numerical calculation, distances $0.3 \mathrm{~m}$ and $0.75 \mathrm{~m}$, respectively; experiment: - - measured by the first channel, distance $0.3 \mathrm{~m}$ from the generators; $\bullet-$ measured by the second channel, distance $0.75 \mathrm{~m}$ from the generators (Fig. $5, \mathrm{~b}$ ); - measured by the second channel, distance $\sim 170 \mathrm{~cm}$ from the generators. (Fig. 5, a).

The time dependence of the aerosol particle concentration in the vertically configured $\Gamma$-shaped chamber at different placement of the aerosol generators is depicted in Figure 9. 


\section{EPJ Web of Conferences}

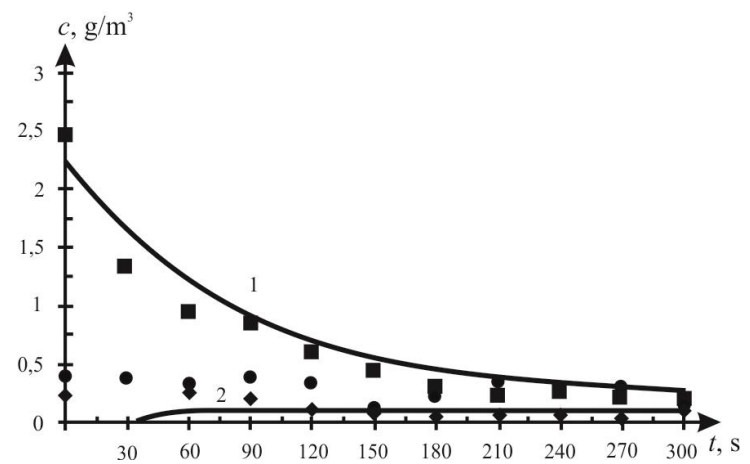

Figure 9. The particle concentration versus time upon propagation in the horizontally configured $\Gamma$-shaped chamber: $1,2-$ numerical calculation, distances $0.3 \mathrm{~m}$ and $0.75 \mathrm{~m}$, respectively; experiment: - measured by the first channel, distance $0.3 \mathrm{~m}$ from the generators (directly above the generators, Fig. 6); $\bullet-$ measured by the second channel, distance $\sim 170 \mathrm{~cm}$ from the generators (Fig. 6 , a); $\bullet$ measured by the second channel, distance $\sim 170 \mathrm{~cm}$ from the generators (when atomized from the top) (Fig. 6, b).

\section{Conclusion}

The experimental and calculated data (for $D=1.2 \cdot 10-3 \mathrm{~m}^{2} / \mathrm{s}$ ) of the aerosol cloud propagation in the confined space of $\sim 2 \div 8 \mathrm{~m}^{3}$ by volume show that both upon the vertical and upon horizontal propagation of the fine aerosol, the concentration range in the confined space becomes homogeneous in $30 \mathrm{~s}$, which is consistent with the diffusion model of aerosol propagation.

\section{Acknowledgements}

The work was accomplished with support from the RFBR grant No.15-38-50660 (mol_nr).

\section{References}

1. N.V. Korovina, O.B. Kudryashova, A.A. Antonnikova, B.I. Vorozhtsov, Izvestiya Vuzov: Fizika, 56 (9/3), 169 (2013)

2. O.B. Kudryashova, N.V. Korovina, A.A. Pavlenko, V.A. Arkhipov, V.D. Gol'din, E.V. Muravlev, Inzhenerno-fizicheskiy Zhurnal, 88 (3), 552 (2015)

3. D.G. Pazhi, V.S. Galustov, Fundamentals of liquid atomization techniques (Protsessy i Apparaty Khimicheskoi i Neftekhimicheskoi Tekhnologii. M.: Khimiya, 1984)

4. V.A. Arkhipov, S.S. Bondarchuk, M.Ya. Yevsevleev, I.K. Zharova, A.S. Zhukov, S.V. Zmanovskiy, E.A. Kozlov, A.I. Konovalenko, V.F. Trofimov, Inzhenerno-fizicheskiy Zhurnal, 86 (6), 1229 (2013)

5. O.B. Kudryashova, A.A. Pavlenko, B.I. Vorozhtsov, S.S. Titov, V.A. Arkhipov, S.S. Bondarchuk, E.A. Maksimenko, I.S. Akhmadeev, E.V. Muravlev (Photodetectors. Rijeka, Croatia: InTech, 2012) 